\title{
Scenario-Based Requirements Engineering Facilitating Interaction Design
}

\author{
Hermann Kaindl \\ Vienna University of Technology, \\ Gußhausstr. 27-29, A-1040 Vienna, \\ Austria \\ kaindl@ict.tuwien.ac.at
}

\begin{abstract}
When the requirements and the interaction design of a system are separated, they will most likely not fit together, and the resulting system will be less than optimal. Even if all the real needs are covered in the requirements and also implemented, errors may be induced by human-computer interaction through a bad interaction design and its resulting user interface. Such a system may even not be used at all. Alternatively, a great user interface of a system with features that are not required will not be very useful as well.

Therefore, the primary motivation of this tutorial is to improve system development in practice both regarding requirements engineering and interaction design, especially facilitating the latter. We argue for combined requirements engineering and interaction design, primarily based on usage scenarios in the sense of sequences of actions aimed at accomplishing some task goal. However, scenario-based approaches vary especially with regard to their use, e.g., employing abstract use cases or integrating scenarios with functions and goals in a systematic design process. So, the key issue to be addressed is how to combine different approaches, e.g., in scenario-based development, so that the result is an overall useful and useable system. In particular, scenarios are very helpful for purposes of usability as well.
\end{abstract}

Keywords: Interaction design, usage scenarios, requirements engineering, user interfaces, usability.

\section{Tutorial Goals}

This tutorial is targeted towards people who are supposed to work on the interaction design or the requirements in systems development, e.g., interaction designers, user interface developers, Web designers, requirements engineers, or project managers. Whatever the roles of the tutorial participants actually are in their daily work, they should get a better understanding of "other" viewpoints and tasks and, in particular, a common approach. The overall goal of presenting this proposed tutorial is to teach how requirements engineering and interaction design relate and how they can be usefully combined. This can be important for creating better interactive systems in the future. 


\section{Key Learning Outcomes}

In this tutorial, participants learn about an approach to scenario-based requirements engineering that facilitates interaction design. In particular, participants will understand how scenarios and use cases can be utilized both for requirements engineering and interaction design, though with different emphasis on the level of detail. They will also understand the additional need to specify the functional requirements for the system to be built, even in the context of object-oriented (OO) development. Overall, they will gain a better understanding of early systems design.

\section{$3 \mathrm{CV}$ of the Presenter}

Hermann Kaindl is the director of the Institute of Computer Technology at the Vienna Univ. of Technology. He joined this institute in early 2003 as a full professor. Prior to moving to academia, he was a senior consultant with the division of program and systems engineering at Siemens AG Austria. There he has gained more than 24 years of industrial experience in software development and human-computer interaction. $\mathrm{He}$ has published four books and more than a hundred papers in refereed journals, books and conference proceedings. He is a Senior Member of the IEEE, a Distinguished Scientist member of the ACM, a member of the INCOSE and the AAAI, and is on the executive board of the Austrian Society for Artificial Intelligence.

\section{References}

1. Kaindl, H.: A Practical Approach to Combining Requirements Definition and ObjectOriented Analysis. Annals of Software Engineering 3, 319-343 (1997)

2. Kaindl, H.: A Design Process Based on a Model Combining Scenarios with Goals and Functions. IEEE Transactions on Systems, Man, and Cybernetics (SMC) Part A 30, $537-$ $551(2000)$

3. Kaindl, H.: Adoption of Requirements Engineering: Conditions for Success. In: Proceedings of the Fifth IEEE International Symposium on Requirements Engineering (RE 2001), invited State-of the-Practice Talk, Toronto, Canada, pp. 156-163. IEEE, Los Alamitos (2001)

4. Kaindl, H.: Is Object-oriented Requirements Engineering of Interest? Requirements Engineering 10, 81-84 (2005)

5. Kaindl, H.: A Scenario-Based Approach for Requirements Engineering: Experience in a Telecommunication Software Development Project. Systems Engineering 8, 197-210 (2005)

6. Kaindl, H., Jezek, R.: From Usage Scenarios to User Interface Elements in a Few Steps. In: Proceedings of the Fourth International Conference on Computer-Aided Design of User Interfaces (CADUI 2002), Valenciennes, France, pp. 91-102. Kluwer Academic Publishers, Dordrecht (2002)

7. Kaindl, H., Kramer, S., Hailing, M.: An Interactive Guide Through a Defined Modelling Process. In: People and Computers XV, Joint Proceedings of HCI 2001 and IHM 2001, Lille, France, pp. 107-124. Springer, London (2001)

8. Kaindl, H., Svetinovic, D.: On confusion between requirements and their representations. Requirements Engineering 15, 307-311 (2010) 